\title{
Publisher Correction: Just astronomy
}

Jörg Matthias Determann (D)

Correction to: Nature Astronomy https://doi.org/10.1038/s41550-021-01313-3, published online 16 April 2021.

In the version of this Books \& Arts article originally published, the image of the book cover was missing; it has now been added.

Published online: 12 May 2021

https://doi.org/10.1038/s41550-021-01390-4

Springer Nature Limited 2021 